\title{
EL RETORNO DE LO CAMPESINO: \\ UNA REVISIÓN SOBRE LOS ESENCIALISMOS

$$
\text { y heterogeneidades en la antropología }
$$

\author{
JAIRO TOCANCIPÁ-FALLA \\ Departamento de Antropología, \\ Universidad del Cauca, Popayán \\ jtocancipa@unicauca.edu.co
}

\begin{abstract}
Resumen
A REVISIÓN CRÍTICA DE LOS CONCEPTOS EMPLEADOS EN LA ANTROPOLOGÍA ES IMportante en la producción de conocimiento. Este artículo examina el caso del concepto campesino, cuestionado por distintos académicos, especialmente en lo que podría llamarse una antropología de la(s) metrópoli(s). La crítica analizada procede de un trabajo reciente del antropólogo Michael Kearney, quien sugiere la sustitución del concepto campesino por otro más elaborado, polybiano, que manifiesta la condición actual de poblaciones rurales que ya no son estables en sus lugares de origen. El artículo plantea que si bien este es el caso de México, no es posible hacer extrapolaciones a otras regiones de América latina, donde tal categorización puede no ser pertinente.
\end{abstract}

Palabras Clave: campesino, polybiano, México, Cauca, antropología.

\section{Abstract}

CRITICAL REVIEW OF CONCEPTS USED IN ANTHROPOLOGY IS AN IMPORTANT ASPECT in the production of knowledge. This essay examines the case of peasant as a concept whose usefulness has recently been questioned by a number of scholars, especially from within a type of anthropology emanating from the metropolis. The critique analyzed here stems from the recent work of Michael Kearney, who has suggested substituting it for the term polybian, which addresses the current condition of rural populations that no longer remain in their place of origin. The essay argues that while this may be the case for Mexico, this cannot be extended to other parts of Latin America, since such a category may not adequately describe these populations.

KEY wORDs: Peasant, polybiano, Mexico, Cauca, anthropology.

\author{
Revista Colombiana de Antropología \\ Volumen 4I, enero-diciembre 2005, pp. 7-4I
}


D ESDE HACE ALGUNOS AÑOS SE HA VENIDO CUESTIONANDO EL TÉRMINO campesino, y se ha llegado a plantear que es una categoría que debería desaparecer, pues no representa la(s) realidad(es) sociales contemporáneas del grupo que intenta representar. Aun cuando la crítica no es nueva, como bien lo sintetiza Kearney (I996) en su trabajo Reconceptualizing the peasantry, los conflictos, las movilizaciones, los desplazamientos, las migraciones nacionales e internacionales, los productores que se vinculan a mercados globales, etcétera, han llevado a la revalorización del término campesino, que por lo general se refería a unas condiciones estables o fijas. En el trabajo de Kearney se cuestionan los esencialismos para dar paso a las hibridaciones y las heterogeneidades que se presentan en un mundo que cambia cada vez más. Si bien su planteamiento teórico se presenta a partir de la realidad mexicana, cabe preguntarse si una situación similar se puede extender a otros casos de América latina, y, en el caso colombiano, qué perspectivas se podrían sugerir desde el punto de vista teórico en las investigaciones sobre grupos campesinos en un futuro.

Reconociendo la importancia y síntesis teórica del trabajo de Kearney, este artículo ${ }^{1}$ cuestiona parte de su planteamiento teórico, y muestra cómo el término campesino puede constituir aún un concepto importante en muchos ámbitos latinoamericanos, aun cuando en otros pueda exigir su reemplazo. Es necesario que la investigación etnográfica incorpore cierta perspectiva histórica sobre el uso de los conceptos, con el fin de establecer su precisión espacio-temporal, que determinaría, a la vez, sus capacidades y limitaciones de uso. En cierta manera, mi planteamiento tiene que ver con el trabajo de Mintz (I998; 2000), quien sugiere que si bien muchas poblaciones se desplazan a otros continentes y países, otras permanecen. Añadiría que, con mucha frecuencia, los que se van no olvidan a los que se quedan, y viceversa.

Aun cuando Kearney reconoce parcialmente este aspecto en su trabajo, este se orienta a desbordar la "caracterización simple"

I. El autor agradece a los pares evaluadores anónimos por los comentarios críticos al texto y por sus recomendaciones. canzado cierto grado de vencimiento, indicando su renovación con otros conceptos y tipologías. Aquí, el autor al cuestionar o para dar paso a otras tipificaciones más complejas (Kearney, I996: 2I). En este sentido, sugiere que los conceptos ya han al- 


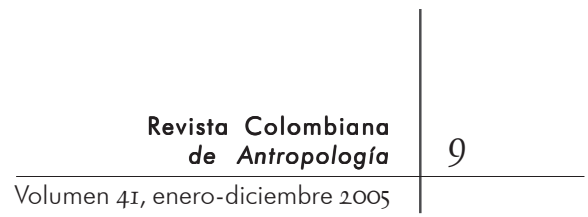

criticar los conceptos termina ofreciendo una salida igual o similar, al retornar a otros esencialismos y tipologías. Mi planteamiento es que el antropólogo exige cada vez más de cierta reflexión crítica frente a la creciente producción literaria y, en especial, a la pertinencia empírica de los términos y a la distinción que exigen. El esquema interpretativo de lo Emic y lo Etic, sugerido por el lingüista Kenneth Pike (citado en Pelto y Pelto, I978: 54), puede ser un marco de referencia distintivo de cómo el mismo término puede analizarse y cuestionarse desde distintos puntos de vista: mientras que para los grupos sociales que se autoproclaman campesinos el término sigue teniendo vigencia, para los antropólogos -en este caso para Kearney- debe ser pensado de nuevo y remplazado, porque las condiciones sociales de esos grupos han cambiado y, sobre todo, porque el cambio de relaciones sociales y de clase implica también la alteración de "los sistemas de signos que define las identidades y valores y, por extensión, las posiciones de clase de los sujetos que los poseen y no los poseen” (Kearney, 1996: 172).

Este artículo se desarrolla en cuatro secciones. En la primera presento una aproximación a la genealogía del término campesino, ejercicio exploratorio que permite conocer las diferencias y similaridades que asocia el concepto en el ámbito de una antropología en general. Es claro que la genealogía o el "pensar genético" tiene raíz en la filosofía, en el trabajo de filósofos como Nietzsche (1994) -por ejemplo, genealogía de la moral, de lo bueno, lo malo-, que valora el espíritu histórico más allá de una simple historia y busca un "buceo en el ser del hombre como ser histórico” (Ferrater Mora, I994: I445). El uso de este término genealógico en este escrito es más modesto, y las pretensiones filosóficas de incorporar un vasto campo de la experiencia humana no son su objetivo. Se podría afirmar que en el caso que nos ocupa, el trabajo de un pensar genealógico se ubica más en el plano de la hermenéutica -literalmente "expresión de un pensamiento" o interpretativo- del término campesino y su correspondencia con lo empírico o la experiencia social que la vincula.

En la segunda examino la idea de lo campesino cuestionada en el trabajo de Kearney, observando particularmente el análisis de las tipologías y la sustitución del concepto campesino por el de polybian. A la luz de algunos trabajos previos sobre los estudios con sociedades campesinas, se trata de esbozar cómo la definición de tipologías sigue siendo una de las preocupaciones 


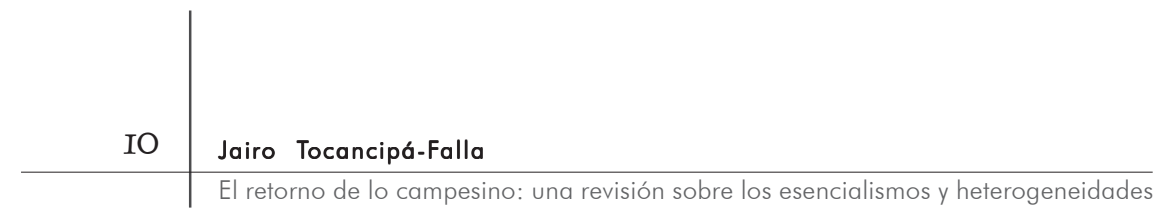

teóricas en la disciplina, a pesar de la crítica que plantea cada una de las clasificaciones presentadas en el pasado.

En la tercera sección presento experiencias en el departamento del Cauca (Colombia), que sirven de ejemplo acerca de cómo el concepto campesino sigue teniendo prelación y pertinencia en procesos de identidad política, por mencionar un problema particular. Concluyo con una parte que destaca la necesidad de seguir haciendo investigaciones etnográficas en colaboración con los grupos sociales con los que interactuamos.

\section{LO CAMPESINO:} GENEALOGÍA(S) DE UN TÉRMINO

ESCRIBIR LA GENEALOGÍA DE LO
tropológica es difícil, porq
fuentes secundarias son varion
ciones etnográficas de las socie
el primer tercio y mitad del siglo
linck, I982; Silverman, I979), y
que se la concedía como grupo
diales ${ }^{2}$. Esto es más claro en el
ocurrida en Europa, en el que
"[...] sólo desde la segunda gue
principales naciones mayorita
2. Según Amerlinck (I982: 33 ), "el estudio sistemá-
tico del campesinado" empezó a tomar forma en
el análisis antropológico "hace unos cuarenta años,
aunque de hecho antes se hayan investigado po-
blaciones en realidad campesinas, como fueran
las sociedades 'Folk'". Si se tiene en cuenta la
fecha de publicación del artículo, serían un poco
más de sesenta años.

científica y, así mismo, fijarían a los países del tercer mundo como la fuente de la producción del conocimiento (Pletsch, I98I). Aún hoy se reconoce que Latinoamérica sigue siendo un laboratorio social no sólo para académicos foráneos sino también para los locales (Escobar y Álvarez, I992).

El interés por estas regiones se despertó, inicialmente, por la imaginería civilizada que existía sobre las mismas. Algunos sectores y 


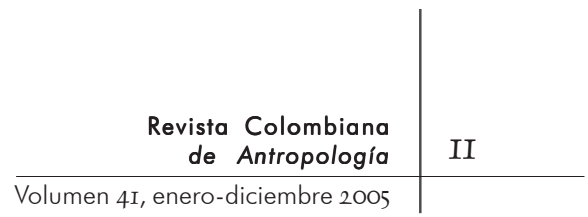

autoridades estadounidenses percibían a América latina, por ejemplo, como una región inferior y que no ameritaba "serias consideraciones políticas e intelectuales”, opinión consecuente con una visión extendida en Estados Unidos, donde América latina era considerada un área poblada "por razas inferiores y dictadores primitivos" (Skidmore, I998: I07), proyecto sugestivo en aquel periodo para una disciplina que en las metrópolis empezaba a verse transformada por la transmutación de su “objeto de estudio". Esta concepción fue consecuente también con el tipo de población que habitaba las zonas rurales de latinoamérica a mediados del siglo veinte. Nombres genéricos como indígenas, mestizos, negros o campesinos abarcaban el amplio espectro de poblaciones que habitaban lo rural. Tales visiones se expresaron en estas categorías conceptuales y en especial en las tipologías que se crearon a partir de las investigaciones, hechas, sobre todo, por antropólogos estadounidenses. En las siguientes secciones se analizarán los aspectos conceptuales y semánticos que mediaron en los análisis de las sociedades campesinas, las tipologías que se formaron y las tendencias investigativas que se derivaron de ellas.

En mi criterio, en la producción de conocimiento antropológico estas consideraciones teóricas deben considerarse críticamente, en especial en relación con el uso de los conceptos que pueden deducirse de las diversas condiciones regionales presentes en los países. Esta sección se concentra en los aspectos de internalidad (cfr. Llobera, I980) que rodearon a los estudios de las sociedades campesinas para el caso de América latina. Mi punto de partida son algunos estudios y referencias académicas de sociedades campesinas hechos por antropólogos y académicos de otras disciplinas.

En un trabajo inicial (Tocancipá-Falla, 1998) sobre los estudios de sociedades campesinas en Colombia en un periodo particular, I940-1960 ${ }^{3}$, discuto los aspectos de internalidad -problemas derivados de la disciplina-, externalidad -factores externos que 3. Un segundo análisis para el periodo subsiguien te, 1960-2004, todavía está por escribirse. afectan o influyen en los problemas de la disciplina- que se daban con este tipo de estudios. Hoy existe cierta conciencia sobre la relación entre producción de teorías, conceptos y los referentes espaciales desde los que los antropólogos las elaboran. Tal como señalaba Fabian (1983), la representación del otro implicó tanto un aspecto de orden temporal como espacial. Esto es claro 
cuando se intenta caracterizar a los grupos campesinos y a lo que en un orden espacial se asocia con el campo, y que por dicha especialidad asocia una condición de permanencia en el tiempo.

No obstante, en muchos países europeos es difícil encontrar, incluso en la actualidad, referencias a poblaciones asociadas a la categoría campesino(s), aunque la expresión siga viva todavía, seguramente para ser usada en grupos de países tercer mundistas. Sin embargo, descriptiva y analíticamente el término tiene su genealogía en el llamado mundo occidental. En el mundo designado como clásico, por ejemplo, los campesinos surgen de manera anecdótica, describiéndolos como un "tipo ideal", asociado a lo saludable, lo simple, sobre todo en la literatura clásica. Así mismo, el "ethos campesino de autosuficiente llamó la atención de escritores clásicos de la elite como un ideal moral, surgiendo con frecuencia en tratados de agricultura [...] Los campesinos y tipos similares fueron romantizados notablemente en la poesía pastoral del periodo helenístico, aunque esta tradición continuó en los tiempos romanos" (Hornblower y Spawforth, [1949] 2003: II30). En esta misma dirección, Wolf (en Stacul, 2000/200I: 66) sugiere que "el término inglés para 'campesino', peasant, viene de paganus, refiriéndose primero al habitante de una sección rural o pagus, y más tarde a no cristianos, pagano”. No obstante, el término peasant procede del francés, que lo asocia con una categoría también espacial, pays, que refiere a país en español. Para ser más precisos, la etimología del término peasant viene de la expresión paisant -de la raíz pays, país- y también rusticus y hombre del campo, para referirse a quienes "trabajan la tierra y viven en el campo" (Williams, I976-I983: 23I). En este caso el uso del vocablo peasant es un ejemplo típico de la influencia de lenguas como la francesa en la anglosajona. Una variación más contemporánea extendida en Estados Unidos es la de farmer, que deviene de farm, granja, heredad, labranza, hacienda.

Existen referencias similares en otras lenguas como en el caso del alemán, en la que campesino es landlich y se asocia también a la tierra. Es interesante destacar la asociación espacial similar con el italiano, en la que campesino es contadino y refiere a alguien que labora la tierra, que es rudo, pero, especialmente en un sentido espacial, se trata de alguien que proviene del contado o "territorio en torno a la ciudad" (Cusatelli, I965: 433). En el caso del español, el campesino(na) refiere a "lo que es propio del campo o perteneciente a él. //2. Dícese de la persona que 


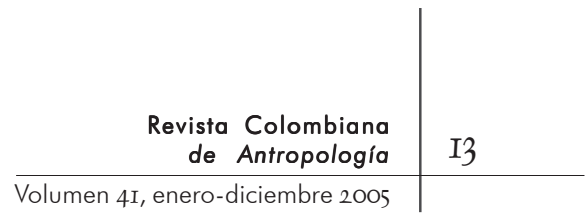

vive y trabaja de ordinario en el campo. //3. Silvestre, espontáneo, inculto" (Real Academia Española, 1992: 380).

En el marco de estas denominaciones dadas en distintas lenguas, el punto por señalar es cómo existen profundas similitudes, que indican la localización de un grupo humano dentro de un espacio y su identificación en carácter y modo de ser, aspecto que será reivindicado por la producción antropológica en general. Dichas denominaciones parecieran indicar que las sociedades campesinas como tipología fueran una constante histórica. Sin embargo, los cambios históricos en las economías condujeron a cambios sustanciales de tales grupos humanos. Williams (I976-I983: 232), por ejemplo, sugiere que "las transformaciones sociales y económicas en la agricultura inglesa del siglo XVI al XIX crearon una dificultad especial para el uso de este término”. En consecuencia, a finales del siglo dieciocho fue remplazado a partir de las nuevas relaciones capitalistas que se expresaron en nuevos términos tales como terrateniente, arrendatario y trabajador agrícola. Cuando dichas transformaciones no llegaron o se asimilaron de manera distinta, los campesinos prevalecieron todavía, conservando la huella del carácter o del modo de ser del campo con el espacio o lugar donde continuaron habitando. Así, por ejemplo, en Francia el término se sigue empleando todavía (cfr. Jenkins, 1994) ${ }^{4}$.

En general, la noción de lo campesino sigue vinculando un sistema axiológico en el que su uso exagerado ha llevado al significado de "gente poco educada" o "gente del común”. No obstante, su uso también ha tenido distintas asociaciones. Williams (I976-I983: 232) indica que el uso del término campesinado en países del tercer mundo to-

4. Esto puede observarse y escucharse todavía en regiones limítrofes con España, como Cataluña donde se refieren a los campesinos como paisanos. davía "lleva un sentido asociado a un grupo social y económico distinto. A lo campesino le han sido dadas, en algunos contextos, connotaciones tanto descriptivas como revolucionarias heroicas". Esta última caracterización seguramente se deriva de la importancia de lo(s) campesino(s) en muchas revoluciones del siglo veinte, como en el caso de México, China, Bolivia y Cuba (cfr. Wolf, I987b). Esta importancia revolucionaria fue asignada inicialmente por Marx, quien consideró a las sociedades campesinas una fuerza revolucionaria, cuando actuaban revolucionariamente, o como una retrógrada, si eran pasivas o anuentes con las instancias de poder: 


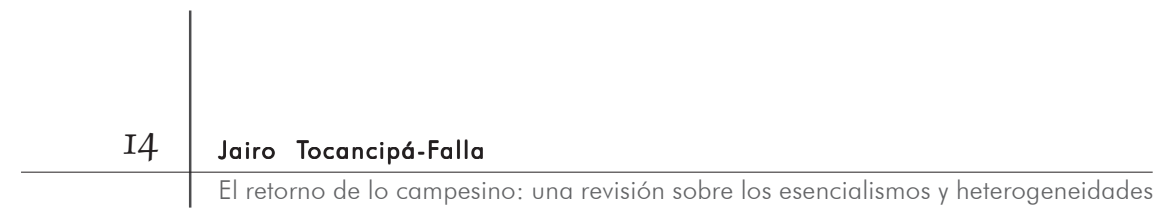

Los estamentos medios -el pequeño industrial, el pequeño comerciante, el artesano, el campesinado [el subrayado es nuestro]-, todos ellos luchan contra la burguesía para salvar de la ruina su existencia como tales estamentos medios. No son, pues, revolucionarios, sino conservadores. Más todavía, son reaccionarios, ya que pretenden volver atrás la rueda de la Historia. Son revolucionarios únicamente por cuanto tienen ante sí la perspectiva de su tránsito inminente al proletariado, defendiendo así no sus intereses presentes, sino sus intereses futuros, por cuanto abandonan sus propios puntos de vista para adoptar los del proletariado (MarX, I973: 120).

La ambivalencia entre actores activos y pasivos empleada por Marx presenta de manera anticipada una de las grandes preocupaciones de la antropología sobre el problema de la tradición y el cambio. Esta tensión constante, todavía por ser investigada en muchas regiones de América latina, tiene varias implicaciones que exceden las posibilidades de análisis en este espacio. No obstante, existen distintos niveles en torno a la genealogía del término campesino, su uso y su posible desuso en las condiciones actuales. Williams (I976-I983: I8-19) hace un análisis interesante de cómo algunas expresiones lingüísticas surgen inicialmente de los registros escritos para pasar luego a lo oral, y cómo otras siguen el camino inverso, de lo oral pasan al reconocimiento de lo escrito. Esta última dirección está vinculada con el referente que le da contenido al término; esto es, el referente empírico que permite evocar aquello que se referencia lingüísticamente.

Es pertinente mencionar la relación entre el uso del término campesino en el contexto académico y el que hacen en la vida diaria los actores localizados en variados contextos sociales. Wolf (2000/200I: 66) aclara este aspecto diferencial entre lo descriptivo y lo analítico:

Pienso que uno debería distinguir entre los términos que se usan en la descripción y los que se utilizan en el análisis. El término inglés peasant viene de paganus (...) Todos estos son términos descriptivos, y se refieren a condiciones particulares en tiempo y espacio. Hemos tratado de usar el término peasant analíticamente, para especificar actores sociales que a) se reproducen por trabajar la tierra; b) pueden tomar las decisiones productivas para hacerlo; c) dependen de un estado dominante, que los incorpora en el pago de tributos e impuestos a las autoridades conectadas a dicho estado. 


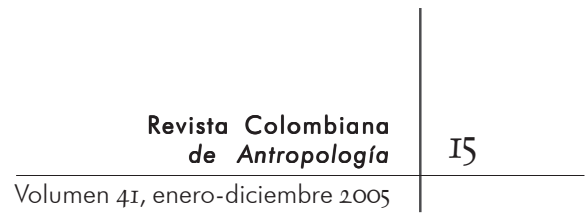

Desde el punto de vista teórico, esta distinción marca una diferencia en el uso de los términos de acuerdo con la perspectiva de quien analiza o interpreta, trátese de quien se considere antropólogo, político, religioso o campesino. Los lingüistas han contribuido mucho en esta reflexión. Vygotsky (I962: I46), por ejemplo, resalta el trabajo de Paulhan, quien establece una distinción entre el sentido de una palabra y su significado:

El sentido de una palabra, de acuerdo con él, es la suma de todos los eventos psicológicos que dicha expresión eleva a nuestra conciencia. Esto es un todo complejo, fluido y dinámico, el cual posee diversas zonas de estabilidad desigual. El significado es sólo una de las zonas de sentido, la zona más estable y precisa. Una palabra adquiere su sentido en el cual aparece. En diferentes contextos, cambia de sentido. El diccionario del significado de una palabra no es más que un ladrillo en el edificio del sentido, no más que una potencialidad que encuentra su expresión diversificada en el habla (...) una palabra en un contexto significa tanto más como menos que la misma palabra aislada: es más porque adquiere nuevo contenido y menos porque el contexto limita y estrecha su significado. El sentido de una palabra, dice Paulhan, es un fenómeno complejo, móvil, de carácter proteico; cambia en diferentes mentes y situaciones y es casi ilimitado. Una palabra deriva su sentido de la oración, la cual a su vez obtiene su sentido del párrafo, el párrafo del libro, y el libro de todos los trabajos del autor.

Esta maravillosa secuencia sobre el sentido de las palabras y su uso de acuerdo con el contexto, permite examinar más detalladamente el análisis que del concepto campesino se ha venido haciendo en distintos contextos en las últimas décadas. Así, la crítica planteada por Kearney (I996), en la que se sugiere cambiar el término peasant por otro más retórico como polybian, invita a reflexionar sobre el contexto que le da contenido a dicha expresión. Aunque se podría reconocer, siguiendo a Vygotsky (I962: I2I-I22), que el "contenido de la palabra no es lo que simplemente cambia sino también el modo en que la realidad se generaliza y refleja en una palabra", considero que el uso de los términos también posee una relación, continuidad y permanencia que se vincula con otras dimensiones en lo político, lo económico y lo social. Es comprensible entonces que Kearney trate de desechar el concepto de peasantry y sustituirlo por otro que refleje con más agudeza la realidad social que estudia. Sin embargo, su invención no es una manifestación del uso social por parte de los actores que experimentan los cambios sociales y 
económicos, ni es parte del proceso de representación que tienen de sí mismos.

Una situación parecida y más cercana puede hallarse en Colombia con fenómenos tales como la violencia, los desplazamientos forzados, las movilizaciones de protesta o los movimientos sociales. En este último caso, por ejemplo, las referencias a lo campesino siguen siendo fundamentales, como ha ocurrido con el movimiento del Comité de integración del macizo colombiano (Cima) (cfr. Espinosa, 1998; Tocancipá-Falla, 2004) que lucha por reivindicaciones sociales desde un punto de vista político. Al igual que el Cima, el término campesino se ha conservado, al menos en muchos contextos de la geografía colombiana, como una expresión política utilizada por los mismos actores para reclamar o reivindicar derechos sociales que no les han sido otorgados por aquello que se denomina el estado, o el gobierno de turno.

Si bien desde un enfoque pretendidamente posmoderno podría cuestionarse el término campesino, por constituir una forma de esencialismo que permite mayor control y poder sobre un grupo de población que el estado requiere administrar, no se puede desconocer tampoco que ese término ha sido empleado también por los mismos actores con el fin, justamente, de legitimizar las demandas que hacen por el cumplimiento de sus necesidades sociales. Desde este punto de vista y desde una genealogía del término, este continúa siendo una expresión importante en cuanto a representación política se refiere. En otras palabras y volviendo a Vygotsky (I962: I53), "las palabras cumplen un papel central no sólo en el desarrollo del pensamiento sino también en el crecimiento histórico de la conciencia como un todo. Una palabra es un microcosmos de la conciencia humana". Estos elementos de la conciencia humana todavía operan en muchos grupos sociales localizados en el área rural, como mecanismos de acción y movilización mediante los cuales se busca cierta reivindicación política.

En resumen, a pesar de cierta singularidad del término campesino, que evoca vínculo(s) espacial(es) y moral(es) con el campo, no existe una sino muchas genealogías del término, enmarcadas en las relaciones sociales establecidas históricamente por los grupos sociales con otros grupos y, en especial, en relación con el espacio donde dichas relaciones se instituyen. Los antropólogos, como otros investigadores sociales, se preocupan por entender y, también, por transformar las condiciones sociales de las denominadas sociedades campesinas. En ese proceso de 


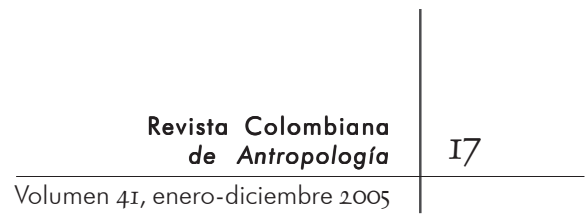

comprender y cambiar puede haber coherencias o incoherencias conceptuales que expresan el ámbito de lo académico escrito y lo oral en distintas formas o modos. Así, los académicos pueden establecer marcos de referencia para explicar las condiciones cambiantes de esas sociedades en términos que no necesariamente se expresen en la cotidianidad de esos grupos pero que pueden expresar, eso sí, las condiciones cambiantes a las cuales se ven sujetos.

El trabajo de Kearney (1996) en el que analiza el caso de México podría verse en esta perspectiva: como una tendencia que señala que el uso del término campesino debe revisarse. Este autor acuña el término polibyan, que aun cuando como se dijo no es utilizado por los mismos actores sí podría explicar muy bien la condición de quienes viviendo en el campo se mantienen desplazándose en distintos contextos: de allí poli, varios, bian o bio, medios o ámbitos. Este aspecto, que podría aplicarse para algunas regiones del país, no necesariamente se convierte en una generalidad. Podría pensarse en los desplazamientos forzados que ocurren en muchas regiones de Colombia, pero también es necesario recordar que las regiones viven en distinta intensidad el fenómeno y hoy resulta complejo y difícil pronosticar qué puede ocurrir en el futuro. Por otro lado, la crisis histórica, social y política en la que se ha visto involucrado el sector rural permite apreciar la importancia del término campesino, como vimos en el breve ejemplo de un movimiento social, en el que su uso también puede constituirse en una estrategia política y cultural, situación que no es exclusiva del Cima.

En conclusión, el conocimiento sobre los mundos campesinos o el/los campesinado(s) (peasantries) en el contexto académico, escrito y oral, demanda más investigación sobre las distintas genealogías que se tienen del término en un contexto en particular ${ }^{5}$. Teniendo en cuenta estas consideraciones, en la siguiente sección se analizan las tendencias y aproximaciones tipológicas y conceptuales ela-

5. Aquí entran en juego las formaciones disciplinarias y lo que se denominan las condiciones de la realidad social que se analiza. En el contexto de la investigación social, sería interesante apreciar cómo entran en juego las distintas representaciones que se tienen de los grupos sociales e, igualmente, los análisis hechos por esos grupos sociales de las representaciones de los académicos.

boradas por académicos esta-

dounidenses para el caso de América latina, y que manifiestan aspectos de orden regional articulados con las formulaciones teóricas que de ellas derivan. 


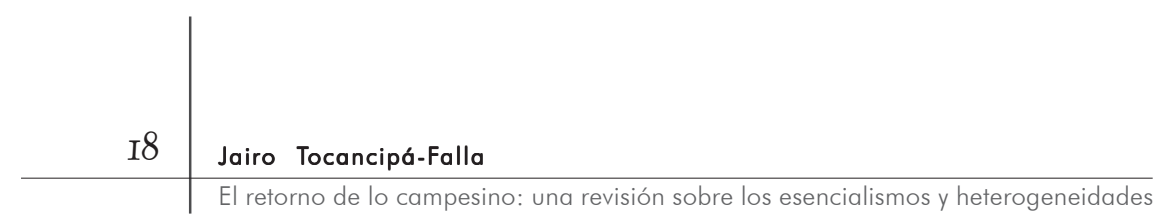

Tipologías EN LOS ESTUdios CAMPESINOS ${ }^{6}$ : ENUNCIACIÓN Y PERTINENCIA

$\left[\begin{array}{l}\mathrm{N} \\ \mathrm{b}\end{array}\right.$ N UN SENTIDO AMPLIO Y CONCEPTUAL, TIPOLOGÍA DERIVA DEL VOCAblo griego typus, o tipo, que significa "marca dejada por el golpe”, "sello", "figura”, "molde”, "impresión”. Existen varias formas tipológicas, que a pesar de sus variaciones comparten un sentido común: "la de hacer posible la comprensión de las individualidades humanas y de sus relaciones con el espíritu objetivo y los valores, sin que ello equivalga a suponer que en la realidad existen los tipos puros". (Ferrater Mora, I994: 35I23516). En el caso de las disciplinas sociales, uno de los ejemplos más conocidos es el de los tipos ideales de Max Weber, el cual lo vincula con el "método científico" sociológico, que según él consiste en "la construcción de tipos" y en el análisis y exposición de "todas las conexiones de sentido irracionales, afectivamente condicionadas, del comportamiento que influyen en la acción, como desviaciones de un desarrollo de la misma 'construido como puramente racional con arreglo a fines”'. Según él, estas definiciones están en correspondencia con el papel interpretativo que la sociología se propone en la investigación de los distintos dominios de la realidad social, sea esta de tipo económico, religioso, político, etcétera (Weber, I944: 6, 8).

El establecimiento de tipologías no fue ajeno a los antropólogos, especialmente en Estados Unidos. Aun cuando es cierto que los estudios sobre sociedades campesinas se asociaron con los estudios folk en el siglo diecinueve en Europa,

6. Una reseña más completa sobre la tipología y los aspectos conceptuales en los estudios campesinos se encuentra en Kearney (I996) y Roseberry (1989a). Aunque aquí se sacan conclusiones similares a los de estos autores, lo que intento es complementar el análisis, especialmente en relación con el trabajo de Kearney, quien no se refiere a los trabajos de Geertz (196I), Wagley y Harris (I955) y Dalton (I972), y especialmente en relación con el trabajo de campo desarrollado en una región del sur del departamento del Cauca.

7. Una revisión crítica puede encontrarse en Lewis ([I95I] 1963).

distintas disciplinas que pueden ser identificados para un periodo similar (cfr. Silverman, I979). Desde la década de I930, su impulso empezó a ser más sistemático en el siglo veinte, especialmente en la década de 1930 con el trabajo de Robert Redfield (I930) Tepoztlan: A Mexican Village ${ }^{7}$. En el caso de los sociólogos, con los de William I. Thomas y Florian Znaniecki, con The polish peasant in Europe and America. Existen, sin embargo, algunos trabajos desde 


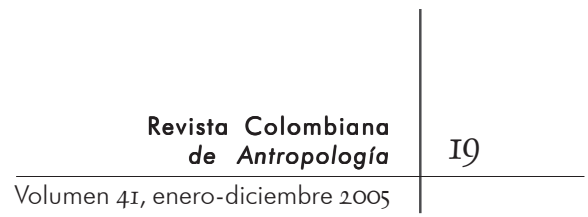

los estudios sobre las sociedades campesinas se han venido incrementando considerablemente.

El artículo "Studies in peasant life”, de Geertz (I96I), constituye uno de los primeros intentos por sistematizar lo que se había escrito sobre las sociedades llamadas campesinas ${ }^{8}$. Su análisis toma en cuenta los trabajos hechos por antropólogos estadounidenses, quienes se dedicaron al estudio de comunidades rurales a mediados del siglo

veinte. Para este efecto, consi- $\quad 8$. Un trabajo previo sobre campesinos antes de dera ciento cincuenta trabajos 1957 es el de Chiva (I958, citado en Geertz, I96r). académicos alrededor del mundo que involucran el concepto de lo campesino, y sus principales tendencias y enfoques de investigación. Lo característico en este artículo de Geertz es la identificación de dos tendencias, que el llama el enfoque intensivo y el enfoque extensivo. El primero se relaciona con los estudios de comunidad o "estudios micro sociológicos", y el segundo trata sobre "las características comunes y los vínculos que conectan y configuran estos diversos segmentos en, al menos, algo así como una unidad coherente" (Geertz, I96r: I3).

A partir de una amplia investigación empírica, Geertz considera que algunos de estos trabajos enfatizaban en el primer enfoque y otros el segundo, y que en ocasiones había una combinación de ambos. Su argumento parte del trabajo de tres antropólogos que estimularon el campo de los estudios campesinos: Robert Redfield, Julian Steward y Kart Wittfogel. Aun cuando ellos confrontaron diferentes aspectos, tales como el estatus cultural, lo ecológico, lo político y lo económico en sociedades rurales, existían interrelaciones entre ellos en que el campesinado hace "parte de la sociedad, la cultura y civilización (sic) dentro de la cual existen" (Geertz, I96r: 4). Dadas estas condiciones y los análisis que de ellas derivaron, se estableció una tipología y un análisis de los métodos de estudio de dichas sociedades. Estos dos modos de categorizar este grupo social -uno basado en el campesinado en sí mismo y el otro en relación con la caracterización del todo sociocultural dentro del cual este existe- determinaron en su momento una tendencia general en los estudios de los grupos campesinos. Lo que ha variado es la naturaleza de las relaciones establecidas históricamente entre estos grupos y los agentes externos o fuerzas de cambio, internas y externas. 
Sin embargo, la distinción teórica entre "comunidad en sí misma" y "en relación con" otros sistemas sociales, ha sido, al parecer, resultado de las influencias e intercambio de ideas de la primera mitad del siglo veinte, que caracterizó a muchos estudiosos de la Otredad. Silverman (I979), por ejemplo, identifica una interesante genealogía y muestra cómo algunas ideas y conceptos fueron tomados de la antropología funcionalista británica, siendo diseminadas por Radcliffe-Brown, Malinowski y W. Lloyd Warner. El adiestramiento de nuevos pupilos en las escuelas de Harvard y Chicago en los años 1930, en donde se transmitieron algunas de estas ideas, involucró a Redfield, quien se venía ocupando de su trabajo germinal sobre sociedades campesinas. Existe una división teórica con los estudios campesinos representada por Redfield y Steward, la cual asocia, de algún modo, la división establecida por Geertz. Mientras que Redfield estuvo involucrado en el concepto británico de cultura, como un elemento "aditivo e integrador" (Silverman, 1979: 55), Steward lo estuvo más en el contexto de la economía política en la que estaban inmersas las sociedades campesinas. En este caso, Silverman reconoce el trabajo de los pupilos de Steward: Wolf, Sydney, Stanley Diamond, Morton Fried, Elman Service, John Murra y Rufus Mathewson, quienes desarrollaron también de manera crítica el enfoque teórico de su maestro: "Su interés en problemas tales como el desarrollo comparativo del estado, la formación de la nación y las estructuras de parentesco en contextos político-históricos fueron estimulados por Steward pero de manera alguna desarrollados a partir de sus iniciativas" (Silverman, I979: 62). Llama la atención cómo esta apreciación coincide con la de Roseberry (I988), quien en su artículo sobre economía política analiza las distintas vertientes que se agrupan bajo estos dos términos, y cómo esta apreciación riñe con el planteamiento de Ortner (I994), según el cual este enfoque teórico fue característico en la década de I970, y cuya manifestación más clara fue la revista especializada The Journal of Peasant Studies.

La creciente producción de trabajos académicos en los años I940 y 1950 y en décadas sucesivas, condujo a muchos antropólogos sociales a pensar que era hora de clasificar las experiencias con campesinos en tipologías que contribuyeran desde una perspectiva comparativa y teórica a comprender mejor esas sociedades. De acuerdo con la expresión de Wolf, para referirse a las "complejidades de las relaciones que son históricamente variables" 


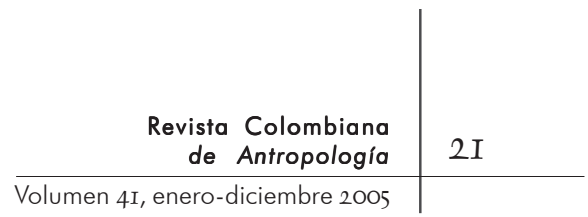

(citado en Friedmann, I987: III) ${ }^{9}$, las tipologías parecen indicar un "lenguaje del tiempo". En términos generales y desde esta perspectiva tipológica, por decirlo así, se pueden identificar los trabajos de Wolf (1955), Wagley y Harris (1955), Geertz (I96I), Dalton (1972), Silverman (I979), Roseberry (I989b) y Kearney (I996). Separados uno del otro por casi una década, en promedio, la mayoría de estos académicos con formación en la antropología basaron sus trabajos de campo en América latina principalmente; Kearney señala la existencia de áreas de poblaciones campesinas no sólo en Latinoamérica sino también en Asia, aun cuando se encuentran bajo la presión de procesos de globalización: “[...] el argumento de este libro es que la mayoría de campesinos se han ido y que las condiciones globales no favorecen la perpetuación de los que permanecen” (Kearney, I996: 3).

Veamos brevemente cómo algunos de estos autores desarrollaron tipologías sobre sociedades campesinas. Wolf, por ejemplo, analiza el concepto de lo campesino basado en autores tales como Redfield y Kroeber, enfatizando en las distintas posibilidades que tienen las relaciones entre las sociedades campesinas y la sociedad como un todo. De esta manera, estudia las transformaciones económicas y sociales en las que dichas sociedades se vieron inmersas ${ }^{10}$ : "Ha habido tipos diferentes de industria y de mercados, de expansión industrial y crecimiento del mercado. Estos han afectado diversas partes del mundo de forma muy distinta. Los campesinados hallados en el mundo de hoy son productos múltiples de tal crecimiento 9. Existe otra clase de tipologías como las de Redfield (I94I) y Steward (I953). Sin embargo, estas son tratadas y sintetizadas por autores posteriores. IO. En esta sección no se analiza el papel político de las sociedades campesinas estudiado por Wolf en otros trabajos posteriores, como en su trabajo clásico Las luchas campesinas en el siglo XX (Wolf, I987b). multilineal" (Wolf, I955: 453). Su principal planteamiento sobre el concepto del campesinado antecede mucho la crítica actual de esencialismos conceptuales, aun cuando cae otra vez en las tipologías que también encierran cierta categorización conceptual. Para él, el campesinado debe ser entendido en términos de "relaciones estructurales y no de un contenido particular [...] una tipología de campesinados debería establecerse sobre la base de regularidades en la ocurrencia de relaciones estructurales más que sobre la de regularidades en la ocurrencia de elementos culturales similares" (Wolf, I955: 454). En este contexto, uno de los factores más importantes por 


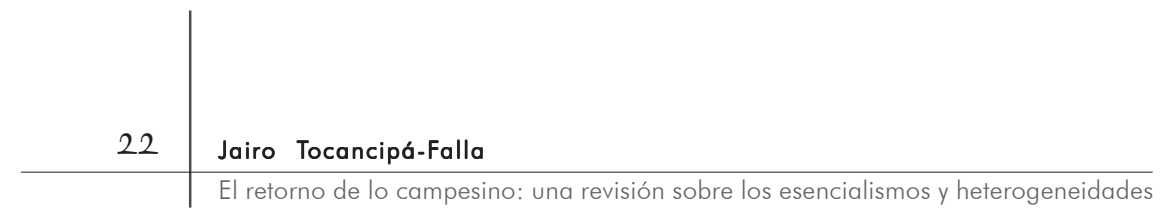

tener en cuenta es la relación de las condiciones sociales del campesinado, los grupos y factores con los cuales interactúan: "Debemos también analizar la manera en la cual ellos están integrados con el mundo exterior. En otras palabras, una tipología de campesinos debe incluir una de los tipos de comunidades en las que viven" (Wolf, 1955: 455). En resumen, este autor señala cinco criterios para la construcción de una tipología. Primero, es necesario delimitar el tema; segundo, debe considerarse un enfoque sobre "la estructura más que sobre el contenido cultural”; tercero, se debe subrayar el criterio económico o sociopolítico, entre otros factores; cuarto, hay que ver los tipos como componentes de partes más amplias o en relación con el todo; y quinto, en este proceso explicativo es fundamental la perspectiva histórica en el análisis.

Teniendo en cuenta estos criterios y basado en algunos elementos de orden geográfico y ecológico, Wolf definió dos tipos en particular: grupos localizados en la parte alta de las montañas de América latina; y grupos en las tierras bajas húmedas y tropicales. Para el primer tipo se asocian aquellas áreas donde prevalecen núcleos de la América colonial española:

Esta puede ser caracterizada por el modo como el individuo se relacionó con el mundo que le rodea. La integración de este campesinado en la estructura colonial se alcanzó por lo general mediante la formación de comunidades que inhibieron el contacto directo entre el individuo y el mundo exterior interponiendo entre ellos una estructura comunal organizada. Esta estructura la llamaremos the peasant corporate community (Wolf, I955: 456; Wolf, I957).

El segundo tipo se refiera a los grupos que "regularmente venden productos agrícolas, que constituyen probablemente entre 50 y 75\% del total de su producción” (Wolf, I955: 46I). Las regiones cafeteras son características de este tipo de campesinado: "El desarrollo de este tipo de campesino ha sido más notable en las tierras bajas húmedas, donde el cultivo estándar es el café [...]. Este es fácil de cultivar tanto en grandes como en pequeñas propiedades, como es el caso de Colombia, Guatemala, Costa Rica, y partes de las Indias Occidentales" (Wolf, I955: 46I). En su momento, Wolf reconocía que este tipo de grupos no había sido estudiado suficientemente por los antropólogos y, por tanto, las explicaciones fueron consideradas parciales. Según él, 


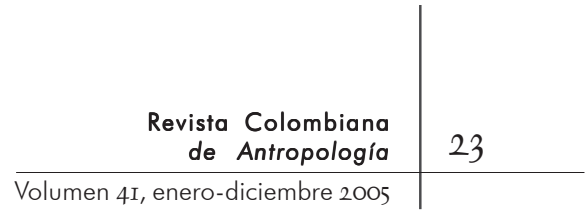

una peculiaridad que distingue este tipo de sociedades de otras sociedades campesinas corporativas es, justamente, la forma abierta como se relaciona con el mundo exterior.

Lo interesante de esta tipología es el reconocimiento de que no agota el amplio rango de sociedades campesinas existentes en América latina. La adición de cinco tipos más al final del artículo y la interrelación entre estos, señala la provisionalidad de su esquema analítico y, sobre todo, ilustra el estado de la investigación sobre este tipo de sociedades en la academia estadounidense en ese entonces. Tres décadas más tarde, Wolf (I986: 326) reconoció las limitaciones de los planteamientos de sus trabajos previos: "lo que estos trabajos muestran es que las interpretaciones muy generalizadas de mediados de los años cincuenta deben ser cualificadas por las variaciones considerables que existen tanto en espacio geográfico como en tiempo histórico". En una de sus últimas entrevistas, se refirió también a las tipologías, y, particularmente, a the closed corporate peasant communities, que "en términos culturales, representa un tipo de campesinado transversal. No estoy utilizando el término analíticamente. Este no es un tipo universal. No todas las comunidades campesinas, pasadas y presentes, son o fueron closed corporate peasant communities" (Stacul, 2000/200I: 66).

En contraste con Wolf, Wagley y Harris (I955: 429) asumen una posición diferente, dando cierta importancia al contenido cultural. Para esos autores, los campesinos son un tipo de subcultura: son llamadas subculturas porque son variaciones de una tradición cultural más amplia y representan una forma de vida de segmentos significativos de la población de América latina. Son llamados tipos porque su contenido difiere de acuerdo con el medio ambiente, la historia y las tradiciones locales, distintas de la nación o la subregión en donde se encuentran. El reconocimiento de variaciones en el campesinado como una subcultura, y también como si compartiera aspectos comunes con una tradición cultural más general, permite sugerir tipos de campesinado. En términos amplios, ellos reconocen nueve tipos de subcultura y hacen la misma advertencia de Wolf acerca de la necesidad de establecer un esquema provisional, demandando en un futuro investigaciones más "refinadas y amplias" sobre tipologías de lo campesino. Sugieren que el proceso creciente de urbanización podría conducir a la extinción y agotamiento de estos tipos de subcultura, pero que, al mismo tiempo, 


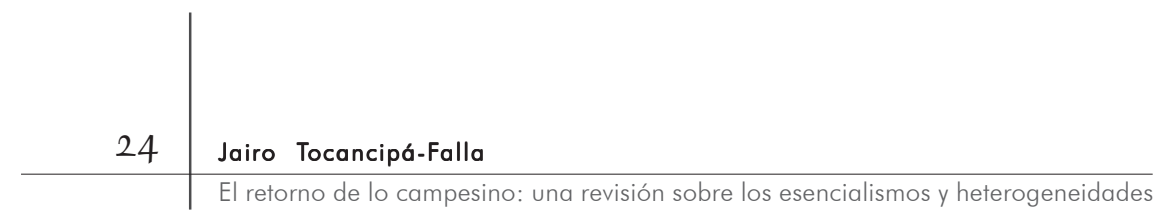

implica el surgimiento de otros tipos que el investigador debe estar en capacidad de reconocer.

Con base en las tipologías de mediados de los años 1950 formuladas en los trabajos de Wolf (1955; 1957) y de Wagley y Harris (1955), se pueden sacar algunas conclusiones. Primero, el reconocimiento de que las investigaciones empíricas demandaban cierto ordenamiento o encasillamiento conceptual en el que las tipologías constituirían el recurso teórico más expedito. Podría afirmarse que la diversidad de modos de vida campesinos, que apenas se empezaba a identificar por medio de las investigaciones, señalaba la necesidad de establecer un sistema clasificatorio que le diera mayor "claridad analítica y conceptual". Contrasta con estas tipologías el comentario de Geertz (I96r: I8), quien en su artículo sobre sociedades campesinas señalaba que "la literatura monográfica sobre comunidades campesinas no es en el momento un lugar apropiado para observar ideas, y ciertamente no para sistemas de ideas".

Segundo, se reconocen las variaciones sociales de lo campesino en América latina, así como el carácter provisional, sujeto al desarrollo de más investigaciones que podrían extender, refinar o revisar mucho más los esquemas conceptuales empleados. Las tipologías de lo campesino plantean así una perspectiva comparativa que apenas empezaba a explorarse. Aun cuando las tipologías planteadas por estos autores, Wolf, Wagley y Harris, también tenían un elemento comparativo, su enfoque estaba enmarcado dentro de los mismos sistemas sociales que lo incluían. Un ejercicio articulador con una visión más general -léase lo global- se vería en las siguientes décadas.

En el artículo de Dalton (1972), "Peasantries in anthropology and history", se intenta superar el ámbito de la región y la comparación de sociedades campesinas en el mundo se hace en una escala mucho más amplia. Sin embargo, su análisis, más condicionado por un modelo marxista de moda, impuso un esquema explicativo lineal evolutivo, que según él contribuiría a comprender mejor a los campesinos. Su perspectiva era más ambiciosa y trató de comparar a los campesinos de ciertas partes del mundo, en especial de América latina, con los campesinos europeos de tiempos medievales: "Estoy sugiriendo que para entender a los campesinados de hoy en la India o Perú es útil estudiar a los siervos europeos en el siglo $\mathrm{X}$ y a los granjeros europeos en el XX, ya que debemos saber en qué cambiaron y en qué cambiarán los 


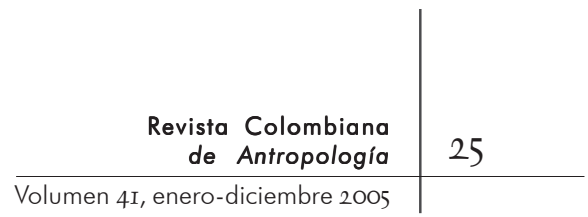

campesinos del tercer mundo (sic)" (Dalton, 1972: 385). Para lograrlo presenta tipos secuenciales de campesinados de Europa occidental, los compara con algunos tipos generales de campesinado -la tipología de Wolf- y, finalmente, con algunos "campesinados de composición híbrida de reciente asentamiento en América latina”. Junto a este esquema lineal evolutivo y a su crítica de los planteamientos del trabajo de Wolf, se halla otro planteamiento, fundado también en un proceso histórico de transformación y que se relaciona con el estado -aspecto que también asocia a Wolf- y otras fuerzas de modernización que concurren en distintos periodos históricos.

Podría afirmarse que la versión de Dalton es un ejercicio comparativo extremo derivado de la década de 1970, justo cuando las perspectivas marxistas y de economía política empiezan a tomar fuerza en la antropología en general (véanse, en este sentido, Marcus, 1995; Ortner, 1994; Sahlins, I976 ${ }^{11}$. En el caso de la antropología y después de la importancia señalada por la llamada II. Roseberry (I997) analiza la importancia del penantropología marxista y la eco- samiento marxista en la antropología y discute las nomía política, el trabajo de críticas que algunos académicos hacen al calificar el pensamiento marxista como una teoría domihistoria (I987a), ejemplifica la relación histórica entre lo local y las fuerzas globales de la economía capitalista. Por las mismas condiciones de interdependencia entre distintos sistemas, cuestiona conceptos claves en la disciplina como el de sociedad. Sherry Ortner (1994) articuló luego su trabajo en una discusión teórica de las tendencias en la antropología, "Theory in anthropology since the sixties". Los comentaristas de este artículo, y en particular Appadurai (I986), plantean el interrogante del problema geográfico, que se elude en el análisis de la antropología teórica planteado por Ortner. Es decir, si bien dichas tendencias teóricas desde los sesenta sugerían interrogantes importantes, también es cierto que dejaban otros sin resolver: ¿en qué regiones geográficas puede aplicarse un enfoque teórico? ¿Es una tendencia general de una antropología que se puede utilizar en abstracto mundial?

Desde los albores de la disciplina, la integración entre lo temporal y lo espacial ya había sido establecida. Fabian (I983: I6), por ejemplo, sugiere que 
[...] el discurso temporal de la antropología, como se formó de manera decisiva bajo el paradigma del evolucionismo, descansó sobre una concepción del tiempo que no era sólo secularizado y naturalizado sino también espacializado por completo [...] los esfuerzos de la antropología para construir las relaciones con su Otro por medio de mecanismos temporales implicaron la afirmación de la diferencia como distancia.

Así las cosas, lo primitivo, lo indígena, adquirían en tiempo y espacio una "condición digna" de ser vinculada dentro del esquema de las metrópolis que imperaba en términos lineales, evolutivos y de poder. Toda sociedad, estudiada o por estudiar, lograría automáticamente una posición en la escala de valores, determinada en los países europeos y en Estados Unidos.

Introducir el problema de escala temporal-espacial permitió anticipar los problemas metodológicos que asocia la investigación antropológica en estos aspectos y, por supuesto, otorgó también importancia a la dimensión espacial, geográfica, relegada ya en las disciplinas sociales y en la antropología (Wallerstein et al., 1996).

En un ámbito disciplinario más amplio, según algunos autores estas líneas de pensamiento produjeron una ruptura epistemológica en la disciplina, cuyos efectos se sienten aún. Roseberry (I989a), por ejemplo, considera que la teoría de la dependencia y de los sistemas-mundo y la economía política acentuaron la crisis en la antropología, aun cuando esta crítica no provino de la disciplina como tal. El centro de estas líneas de pensamiento no fue la localidad o el poblado, sino el contexto global y los procesos históricos de los cuales la localidad como tal es un resultado de esa relación dialéctica con el todo. Esta relación, sin embargo, no es fácil, y como lo señala Roseberry (I989b: III) "El intento por mantener una perspectiva antropológica dentro de un marco global presenta enormes problemas históricos y metodológicos". Si bien reconoce que debemos ser "creativos en

I2. Una discusión más elaborada en lo metodológico sobre la trascendencia de lo local y conectando a lo global puede verse en Marcus (I995). nuestra conceptualización", otorga prioridad al desafío metodológico: "Más importante para los propósitos actuales es una discusión de los estilos metodológicos para el entendimiento de esta complejidad" (Roseberry, I989b: $\mathrm{I} 23)^{12}$. 


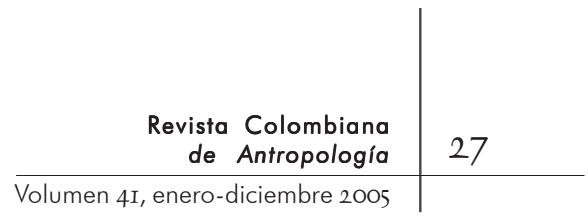

En el orden de las tipologías y de los aspectos conceptuales relacionados con el campesinado, y después de las discusiones de Dalton y Roseberry, Kearney (I996) presenta, quizás, el trabajo más actualizado y representativo de los noventa sobre los estudios de sociedades campesinas: Reconceptualizing the peasantry. Anthropology in global perspective. Retomando la idea de "strategies of containment" de Jameson (I98I) ${ }^{13}$ se interroga, críticamente, sobre el término campesino, que sugiere debe ser descartado, dadas las condiciones actuales a las cuales se enfrenta este tipo de grupo social. Aun cuando cuestiona también algunas de las tipologías que se presentan alrededor de lo campesino y que son complementadas en este artículo, no elude la necesidad de conceptuar las nuevas formas de relacionar a este tipo de sociedades en nuevos ámbitos transnacionales. Recomienda entonces otras expresiones que reflejen mejor los nuevos referentes empíricos de los sujetos que se desplazan en distintos medios y que se encuentran menos relegados a la localidad. Lo polybian, la retícula y las redes acuden como términos sustitutos para describir las nuevas condiciones. El término polybian se refiere a los individuos que viven en distintos contextos y no en un ámbito tan delimitado como implica el término campesino. De allí que polybian deviene en alguien que se "desplaza y vive en distintos medios (poly, muchos + bios, modos de vida)" (Kearney, I996: I4I).

Retícula, redes son tomados de la biología y de la teoría de sistemas para ejemplificar las ramificaciones que se dan dentro de las "naciones, comunidades, otros espacios y cuerpos sociales" (Kearney, I996: I26I27. Véase Deleuze y Guattari, [I980], I997 ${ }^{14}$. Estos dos últimos autores, por ejemplo, introducen el concepto de rizoma, que se entiende a partir de su I3. Las "strategies of containment" hacen referencia a las formas por medio de las cuales en la literatura estadounidense y en los estudios culturales "se proyecta la ilusión de las lecturas como algo autosuficiente y completo" (Jameson, I996: IO); esto es, con un sentido de totalidad que en este caso se extiende al concepto de lo campesino.

I4. Una versión teórica más reciente sobre redes es The actor network theory (ANT). Inicialmente se consideró un "método con implicaciones teóricas" (véanse Boissevain, 1979: 392; Scott, 1996: 794), y más tarde se cristalizó en la forma de ANT. Para una definición y crítica de este marco conceptual véanse Singleton y Michael (1993); Mol y Law (1994); y Latour (I988).

carácter lineal y abierto:

El rizoma conecta cualquier punto con otro punto cualquiera, cada uno de sus rasgos no remite necesariamente a rasgos de la misma naturaleza; el rizoma pone en juego regímenes de signos muy distintos e incluso estados de no-signos. El rizoma no se deja reducir 
ni a lo Uno ni a lo Múltiple [...] No está hecho de unidades, sino de dimensiones, o más bien de direcciones cambiantes [...] El rizoma sólo está hecho de líneas (Deleuze y Guattari [1980], 1997: 25).

La alusión a estos nuevos conceptos se fundamenta en el hecho de que "la mayoría de los campesinos se ha ido y las condiciones globales no favorecen la estabilidad de los que permanecen" (Kearney, 1996: 3). Aun cuando la mayor parte de ese libro es una crítica teórica de la construcción del concepto de lo campesino dentro de la antropología, su análisis y argumentación se fundamentan, principalmente, en el caso mexicano, y en especial en los mixtecas que migran con frecuencia a Estados Unidos como mano de obra o proletarios agrícolas ${ }^{15}$.

El último capítulo del libro considera los aspectos prácticos y teóricos acerca de cómo los mixtecas se relacionan con distintos ámbitos y movimientos sociales que se ocupan de defender los derechos sociales de este grupo indígena que migra a Estados Unidos. De su análisis parece deducirse entonces la extensión de su marco conceptual a otras latitudes donde los campesinos persisten tercamente. Aun cuando el trabajo de Kearney posee aspectos válidos sobre la trayectoria teórica, su horizonte parece estructurarse en un buen caso de lo que tan en boga recientemente se denomina lo global y el transnacionalismo ${ }^{16}$. De este modo, parece que lo global, como un fenómeno social amplio, se hubiera convertido casi en un factor determinista en las relaciones sociales e identidades que diversos grupos sostienen a comienzos del milenio. Mintz cuestiona la novedad aparente de estos argumentos (Mintz, I998: I3I):

Las nuevas teorías del transnacionalismo y la globalización no son suficientemente respetuosas de la historia, en especial de la historia de la exploración, la conquista y la

I5. Una crítica similar al trabajo de Kearney ha sido planteada por Marc Edelman (1999) en su trabajo Peasants against globalization.

I6. Para una crítica sobre el transnacionalismo como materia reciente, véase Mintz (I998).

I7. Para una discusión reciente entre los modelos global-local/folks, véase Holtzman (2004).

\footnotetext{
tradiciones históricas particulares ${ }^{17}$.
} división global del trabajo. Así, olvidando la perspectiva histórica -ia pesar de reclamar que se adopta una perspectiva histórica!- las modas globalistas eclipsan el contexto específico y regional, que provee una base sobre la cual identificar y estudiar problemas de investigación culturalmente específicos, y que tratan con 


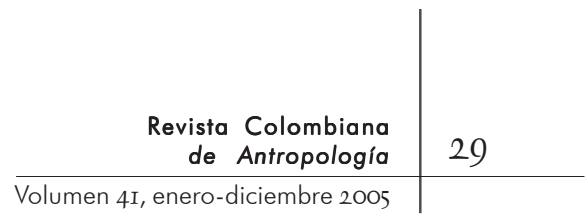

Otro aspecto central en el argumento de Kearney es la posición que ha ocupado lo campesino en la historia de la teoría antropológica: aquí el autor presenta su esquema analítico más detallado a través de las distintas etapas que han caracterizado a la disciplina, empezando desde el periodo formativo -finales del siglo veinte-, y pasando por el clásico -I900-I945-, el moderno -I945-I999- y el global -2000-. Simultáneamente, sostiene que la historia de la clasificación en la antropología y sus tipos básicos ha derivado a lo largo de los últimos cincuenta años de las categorías binarias y esencialistas -lo primitivo- a lo ambiguo -campesinos- y a lo reticular - polibyan-. Aunque en su formulación existen planteamientos interesantes, Kearney termina reproduciendo lo que critica. Es decir, su modelo termina siendo un esquema neo-evolutivo que se desplaza de lo simple a lo complejo, de lo particular a lo global, omitiendo de nuevo aspectos históricos que pueden ser contingentes a los contextos sociales y culturales que trata de comprender y explicar. Más aún, induce a creer que la complejidad es un resultado que antropólogos recientes han sabido identificar y comprender. A pesar de criticar el esencialismo del término campesino, él termina en un sistema de categorías que induce a creer en su carácter esencialista, que se expresa en la "etnicidad del pueblo mixteca": "Este desplazamiento conceptual hace posible integrar cada una de estas identidades locales y sus respectivas formas organizativas -pueblos, sindicatos, organizaciones populares urbanas-, sin perder necesariamente su especificidad local" (Kearney, I996: I85). De acuerdo con esto, los esencialismos étnicos adquieren distinción en el contexto de las condiciones de globalidad que se imponen. Esta ambigüedad o, mejor, paradoja, deja abierta la posibilidad de indagar un poco más acerca de las condiciones históricas regionales o locales sobre las que los antropólogos investigan en distintas regiones, y sobre la pertinencia de los conceptos empleados para su descripción y análisis. A continuación presentaré algunos ejemplos de cómo este término persiste, muy a pesar de las intenciones de establecer su uso inadecuado en el presente. 


\section{"NOSOTROS LOS CAMPESINOS": PERTINENCIA DEL USO DEL TÉRMINO CAMPESINO Y SU RELACIÓN CON EL ENTORNO SOCIAL}

N EDIANTE LA COMPARACIÓN CON LAS GENEALOGÍAS Y TIPOLOGÍAS ESTAblecidas por distintos antropólogos que basaron sus investigaciones en el contexto latinoamericano y desde una perspectiva Etic, es posible establecer las condiciones en las que el término campesino conserva todavía cualidades de identidad, pertenencia e importancia social y política. El departamento del Cauca, situado en el sur de Colombia, abunda en ejemplos según los cuales el uso del término y concepto de campesino es todavía pertinente. Como antecedente es importante señalar que dicho departamento ha sido más rural que urbano; esta distinción problemática se presenta como una constante histórica, considerando incluso fenómenos tan complejos como el desplazamiento forzado y el conflicto armado, que contrasta con las migraciones orientadas y la expansión de los cultivos de coca y amapola.

Las estadísticas estatales indican que desde hace dos décadas la población localizada en las áreas rurales del Cauca corresponde a más de 60\%, mientras que las poblaciones urbanas, localizadas sobre todo sobre el eje de la vía Panamericana, tienen un porcentaje superior a 30\% (Ortega, 200I: 98; Tocancipá, 200I). El departamento se compone de cuarenta y un municipios, con una población total proyectada de I’367.496 habitantes, distribuidos en 30.495 kilómetros cuadrados (Ortega, 200I: 98, 99) ${ }^{18}$. En esta distribución, "la región andina caucana es la más poblada del departamento, con una densidad de población rural alta, 6I a Ioo habitantes por $\mathrm{km}^{2}$, mientras que las regiones de páramo, valles

I8. Dado que en algunas instituciones estatales la referencia a estos datos varía, dichos valores deben considerarse como aproximados. interandinos y costa tienen densidades bajas y medias: I, II y 30 habitantes por $\mathrm{km}^{2}$, respectivamente" (Igac, I997, citado en Tocancipá, 200I: 376). En términos sociales, el Cauca ha sido tipificado como uno de los departamentos del país con mayor diversidad étnica. De acuerdo con Ruiz (200I: 323), cerca de I4\% de su población es indígena, localizándose "en 26 de los 40 (sic) municipios caucanos" y alrededor de $8 \%$ de sus habitantes son afrocolombianos, ubicados en la costa pacífica y los valles interandinos, dejando un margen alto a poblaciones campesinas, colonos y 


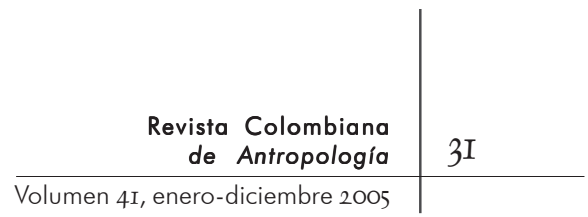

mestizos, localizados también en el área andina y en los valles interandinos.

Más allá de las estadísticas, el cuadro de interacción entre los distintos grupos sociales, incluyendo las sociedades campesinas, puede ser ilustrado en un primer ejemplo relacionado con el caso del Comité de integración del macizo colombiano (Cima). Este movimiento surgió a finales de la década de I980, aun cuando existen antecedentes sobre formas de protesta social reportados en el periódico local desde décadas atrás, como una manifestación de la inconformidad social de las poblaciones del sur del departamento frente a la "política tradicional" y la ausencia de programas sociales consistentes por parte del gobierno regional y nacional. Su conformación se relaciona con la variedad étnica señalada para el caso de la región andina del Cauca, y que tomó como espacio social el gran nudo del Macizo Colombiano: "El Macizo es como una colcha de retazos donde los grupos indígenas, los campesinos, los negros deben encontrar representación” (Walter Aldana, I999, ex líder del movimiento, citado en Tocancipá-Falla, 2004).

Durante las distintas movilizaciones y cierres a la vía Panamericana ${ }^{19}$, la referencia a "nosotros los campesinos" fue permanente, y mediante el uso de esta expresión se enfatizaba en el vínculo con el campo y el suministro de alimentos a poblaciones urbanas. Este énfasis no se apreció sólo en el caso de los movimientos sociales. Durante mi trabajo como representante de un programa auspiciado por una institución no gubernamental a comienzos de los años I990 en el sur del departamento del Cauca, me encontré inmerso en diálogos sobre la problemática de las comunidades rurales. En uno de esos I9. Desde I989 ha habido más de tres movilizaciones, siendo la de noviembre de I999 la más notoria en términos de duración y permanencia en el bloqueo de la Panamericana y el consiguiente aislamiento de Popayán (la capital del departamento). Una de las características más destacadas fue la solidaridad de la mayoría de popayanejos hacia el movimiento, exigiendo la solución a "los problemas de los campesinos". Desde esta última acción, el movimiento no ha presentado otro tipo de acción o movilización destacada. encuentros, un campesino afrodescendiente del valle interandino del Patía me señalaba cómo, "aunque producimos comida, nosotros los campesinos siempre hemos sido olvidados por el estado", en una referencia permanente a la condición de productores o agricultores, relegados históricamente en la política social por parte de esa entidad llamada estado. La política de hacer referencia a un término como el ser campesino se torna 
necesaria en esos casos en los que la identidad o la representación constituyen una estrategia política y de reivindicación social, aun cuando este ámbito no es necesariamente el único.

Otras disciplinas también proveen ejemplos: así, por ejemplo, en relación con la recuperación de la historia regional y local, la formación de los grupos sociales y las relaciones contradictorias que se presentan en una región particular. El alinderamiento de campesinos e indígenas frente a otros grupos sociales dominantes como los terratenientes es otro caso en el que -en términos sociales y de identidad política- el factor diferenciador también se pone de manifiesto. Esta importancia, que se aprecia no sólo desde el punto del investigador sino también desde el de las poblaciones inmersas en ese tipo de relaciones, se puede apreciar en la Historia doble de la costa (Fals Borda, 1986, [2002]). Así, en una primera interpretación del autor o del sociólogo, el académico explica que:

La clásica vocación campesina por la tierra para la utilización de los recursos naturales básicos que tanto abundan en Colombia, especialmente para producir comida, no ha desaparecido con el impacto de la modernidad capitalista ni con el de la nueva tecnología. Hoy mismo en la Costa Atlántica se experimenta un regreso al interés agrario estimulado, como era de esperarse, por campesinos, pescadores e indígenas que siempre han dependido del uso de la tierra, del bosque y del agua para poder vivir (énfasis mío) (Fals Borda, I986 [2002]: I2B).

Junto a esta explicación del investigador o del especialista (canal B), se encuentra la versión de aquellos(as) que se autodefinen como campesino(as) y que se representan mediante el "relato, la descripción, el ambiente, la anécdota” (canal A):

En realidad somos indígenas. Pero nos identificamos con los campesinos en el hecho de que tenemos un enemigo común, que es el terrateniente. Todos luchamos por el mismo derecho y por la misma justicia. Lo que nos mueve a defendernos es nuestro afán compartido de vivir la tierra, nuestras ganas de reafirmarnos como pueblo de productores y vendedores de comida, como agricultores verdaderos que no queremos depender de nadie sino crecer en nuestra finca lo que Dios y nosotros mismos decidamos (Fals Borda, I986 [2002]: 23A) (énfasis mío). 


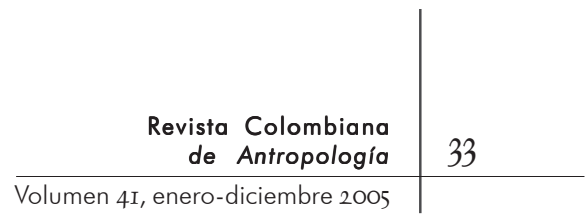

$\mathrm{Al}$ igual que en el caso del movimiento del sur del Cauca y en el del valle del Patía, estas dos apreciaciones validan aún más el hecho de que el uso de los términos o conceptos debe juzgarse en relación, justamente, con el valor relacional, semántico, político o no, que les otorgan las poblaciones. En este orden de ideas, existe cierta congruencia entre la perspectiva Etic y la Emic, que no necesariamente deben darse siempre de este modo. El planteamiento de Kearney de querer dar de baja el término campesino, como sugieren otros académicos, no es, necesariamente, generalizable. Si bien el caso que él analiza puede ser aplicable para una región en el norte de México, deja todavía muchas dudas sobre la pertinencia en otros casos de Latinoamérica (véase Edelman, I999). Como vimos en el caso del departamento del Cauca y del norte del país, el uso del término campesino sigue teniendo vigencia como una forma de representación social en relación con otros grupos sociales y en condiciones políticas, que no las únicas, que ameritan tal identidad.

\section{Notas FinAlES: INVESTIGACIÓN \\ ETNOGRÁFICA Y CORRESPONDENCIA \\ CON MARCOS TEÓRICOS REGIONALES}

S

N ESTE ARTÍ́CULO HE SUBRAYADO LA IMPORTANCIA GENEALÓGICA, LINgüística y etnográfica de lo campesino como mecanismos esclarecedores de su uso, desuso o renovación conceptual. Al igual que muchos conceptos empleados en la disciplina -por ejemplo, cultura, sociedad, indígena, identidad, etcétera-, he sugerido que la revisión crítica del uso de los conceptos es una tarea cada vez más necesaria en la producción del conocimiento antropológico. Esta idea, obviamente, no es nueva, al menos en dos sentidos. Primero, ya ha ocurrido con la crítica de otros conceptos que se consideran insostenibles -por ejemplo, sociedad, cultura e identidad como conceptos pilares de la disciplina-. Segundo, la discusión presentada aquí podría evocar más de la misma que se sostuvo en México entre campesinistas y descampesinistas en la década de I970, que ha adquirido nuevos aires en las últimas décadas y de la cual no escapa la planteada por Kearney. Aunque no presenté conceptualización alguna sobre lo campesino, la intención del artículo es, justamente, no 
anteponer discusiones generadas en otros ámbitos sino favorecer la investigación etnográfica como punto de partida y no como elemento subsidiario en el campo de las definiciones y las representaciones sociales. El caso ilustrado aquí de lo campesino en el ámbito de antropólogos familiarizados con América latina enseña que la revisión conceptual debe basarse en la investigación etnográfica e histórica.

Existen problemas en este sentido. La investigación antropológica que adelantan investigadores sociales de países como Estados Unidos, por citar un ejemplo, permite suponer que los análisis hechos y que se derivan de países o regiones en los que se hallan poblaciones llamadas campesinas aún siguen dedicados al refinamiento de modelos teóricos ya ideologizados. Aun cuando esta no es una generalidad, sí es una tendencia. Estos análisis son hechos por autores que escriben principalmente en inglés, y por lo general existe poca interacción con investigadores locales, la mayor parte de ellos de habla española. Como personas y académicos tenemos diferentes maneras de percibir, y concluyo con tres aspectos que considero se deben observar, aun cuando no son los únicos, en el trazado de la(s) genealogía(s) de los conceptos. Me refiero a las escalas del espacio (lugar), tiempo y a la definición de la(s) preguntas o problema(s) de investigación.

En cuanto a escalas de espacio (lugar), me refiero a las implicaciones teóricas de la investigación etnográfica en la producción conceptual. El uso de los conceptos producidos en las metrópolis sigue teniendo un papel fundacional en las disciplinas sociales en Colombia, como en muchos países de América latina. Aprendemos conceptos de materiales escritos y los reproducimos en la lectura de nuestra realidad(es), aun cuando esta no es, necesariamente, la única dirección posible. Aquí tenemos un problema con el lenguaje que empleamos y desarrollamos a partir de nuestras observaciones. Buena parte de los argumentos de Kearney son bien fundados en la revisión literaria y crítica de algunos conceptos, aunque de nuevo termina magnificando la experiencia mexicana como un caso extensivo a otras regiones de América latina. El problema no es la sustitución de los conceptos, sino en qué medida la investigación etnográfica, históricamente localizada, es suficiente para indicar un giro sustitutivo de esa naturaleza. La importancia del lugar en la investigación adquiere aquí cierta resonancia. Si para Kearney el concepto de lo campesino no es adecuado a la condición 


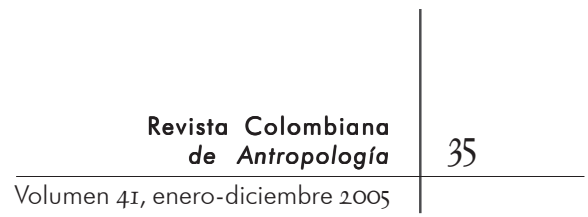

social de los emigrantes mexicanos -otro par de conceptos por discutir- con los que se investiga, entonces es posible preguntarse, ¿̇cuál es la autodesignación, si es que existe alguna, que dichos actores emplean, la visión Emic? Es claro que el uso del término polybiano es una invención del antropólogo que termina dificultando la descripción de los actores con los que nos relacionamos.

Como bien anota él cuando cuestiona a otros colegas, en la crítica de los conceptos se corre el riesgo de seguir la metáfora de que al "botar el agua de la bañera, podemos terminar arrojando al bebé también”. Al igual que el movimiento social del Macizo Colombiano (véanse Espinosa, I998; Tocancipá-Falla, 2004), existen muchos ámbitos donde este término de lo campesino continúa teniendo vigencia, al menos para quienes consideran que hacen parte de esta categoría.

Otra conclusión se puede obtener de este ejercicio genealógico y de tipologías. Me refiero a la escala temporal en la que se insertan tales elaboraciones conceptuales. Desde una perspectiva historicista y de acuerdo con algunos de los trabajos reseñados, la tendencia en el análisis de los grupos campesinos parece oscilar desde la perspectiva esencialista de Wolf, the peasant corporate community, pasando por formas tipológicas que intentan caracterizar las distintas formas y estilos de vida campesinos, hasta los enfoques que presentan lo campesino ya no como entidades sino como sistemas que se establecen en redes en un contexto cada vez más global. Sin embargo, esta orientación de lo esencialista a lo heterogéneo y complejo desde una perspectiva histórica es engañosa también. Existen paradojas en las que tales condiciones no necesariamente son contiguas en un contínuum lineal, sino que pueden ser correspondientes o coexistentes con otras direcciones todavía por cartografiar. Con bastante frecuencia se corre el riesgo de que los análisis puedan terminar en una proyección de los constructos del investigador y la teoría dominante en uso. No se trata de utilizar el esquema analítico de los esencialismos para comprender las sociedades rurales, sino de que mediante el entendimiento de tales grupos en el conjunto de sus relaciones sociales, políticas o económicas -y a riesgo de hacer una separación también artificiosa de estos dominios-, se observe cómo esos aspectos de identidad se manifiestan en espacios de tensión y negociación en distintos niveles y condiciones. Al igual que en la crítica de clase social, la noción de espacio social y campo de 


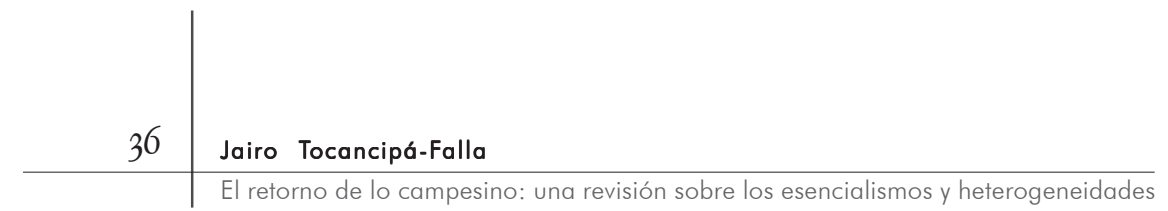

poder plantea problemas similares a los señalados en cuanto a la posición de los actores dentro determinada estructura social (véase Bourdieu, 1994 [1997]).

Aparentemente, he abordado lo conceptual como algo separado de los problemas que agobian a los grupos con los que interactuamos. Sin embargo, esta separación es engañosa, ya que, como se indicó, los términos y conceptos pueden ser utilizados estratégicamente frente a situaciones de conflicto, tensión o negociación, como a veces ocurre con lo campesino. En este orden, la investigación etnográfica concertada con los grupos campesinos ofrece campos interesantes de trabajo tanto teórico como práctico. Así mismo, en este esfuerzo es posible encontrarse con problemas de escala y complejidad ${ }^{20}$, que vinculan a los mismos problemas que han preocupado a los antropólogos y a los grupos en general. Mientras en la década de I940 para muchos antropólogos era importante dar cuenta de la cultura y la personalidad de estos grupos sociales, los gran-

20. Cabe anotar que este aspecto no es nuevo en la antropología, y ya en la década de I980 se planteaban en la disciplina los problemas de escala y complejidad. Véase, por ejemplo, la discusión que Appadurai (I986) y Hannerz (I986) hacen del artículo de teoría antropológica de Ortner (1994). Un ejercicio similar con respecto a Writing Culture puede encontrarse en Fardon (I990). El trabajo de Kearney lo que hace es enfatizar en este aspecto, aplicado para el caso de las sociedades campesinas. sociedades campesinas parece gravitar sobre el conjunto de relaciones sociales, políticas, religiosas, económicas y culturales que estos grupos parecen establecer dialécticamente frente a contextos más amplios. Dos ejemplos recientes son el trabajo de Enrique Mayer (2002), The articulated peasant, y Marc Edelman (I999), Peasants against globalization. En el primero hay una perspectiva desde la antropología y la economía, especialmente en el caso de algunas familias en el Perú que se ven inmersas en relaciones de intercambio con un sistema de mercado más amplio, y particularmente en el contexto de las políticas neoliberales que vive el continente. En el segundo caso, Edelman analiza, desde una perspectiva de la economía política, cómo algunas organizaciones campesinas responden a las políticas gubernamentales nacionales e internacionales y al libre mercado que afectaron en décadas pasadas, sobre todo, al sector agrícola. En mi criterio, estos dos trabajos 


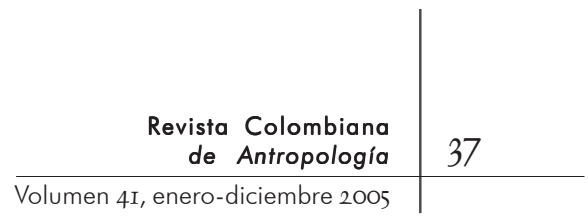

son representativos de un interés creciente por conocer cómo en la actualidad los grupos llamados campesinos reaccionan, resisten, aceptan o transforman sus condiciones en relación con sistemas políticos amplios y otros grupos sociales, políticas, economías nacionales e internacionales que los afectan. Sin embargo, existen otros aspectos, como el religioso, el conocimiento local y la tecnología, entre otros, que también hacen parte importante del estudio de los grupos sociales que llamamos campesinos. Las religiones y los cambios económicos, por ejemplo, son muy importantes, en especial en el caso de América latina, que históricamente se precia de practicar el catolicismo en múltiples manifestaciones.

Este planteamiento sugiere que hay mucha investigación por desarrollar alrededor de lo campesino y su vigencia en relación con el uso de los conceptos empleados por los antropólogos. No existe una genealogía, sino muchas genealogías todavía por ser investigadas. Los tres aspectos que he señalado, espacio, tiempo y problemas de investigación, pueden ser orientadores en este esfuerzo. Como indiqué al inicio del artículo, este es un ejercicio exploratorio desde el punto de vista teórico que exige contenido(s) de investigación(es) etnográfica(s), y la revisión cuidadosa y crítica de la correspondencia conceptual con quienes colaboran en las investigaciones. Más que un punto de llegada es uno de partida, que en el caso nuestro y en medio de los conflictos presentes exige más investigación etnográfica.

\section{Referencias}

AmerlincK, D.B., Marijose. I982. "¿Cultura? ¿Sociedad? ¿Economía? O de cómo la antropología descubrió a los campesinos”. Revista de Anales de Antropología. II. Instituto de Investigaciones Antropológicas.

Appadurai, A. I986. "Theory in anthropology: Center and periphery”. Comparative Studies in Society and History 28. 356-36I.

Boissevain, J. I979. "Network analysis: a reappraisal". Current Anthropology. 20.

Bourdieu, P. [1994] I997. Razones prácticas. Sobre la teoría de la acción. Anagrama. Barcelona.

Chiva, I. I958. "Rural communities: Problems, methods and types of research”. Rep. and Pap. in Soc. Sci. Io (Unesco). 


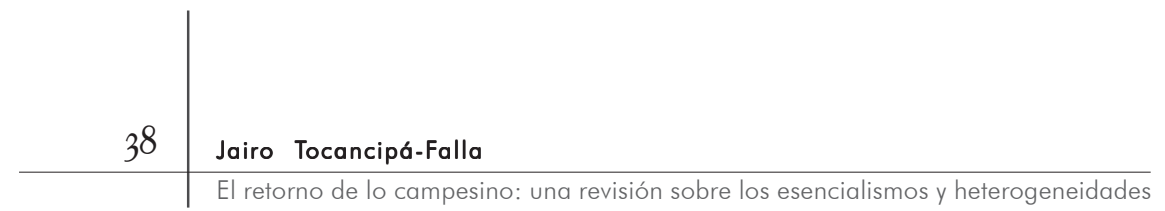

Cusatelli, G. I965. Dizionario Garzanti della Lingua Italiana. Aldi Garzanti.

Dalton, G. I972. "Peasantries in anthropology and history". Current Anthropology. I3.

Deleuze, G. y F. Guattari. [1980] I997. Mil mesetas. Capitalismo y esquizofrenia (trans.) J.V.P.c.l.c.d.U. Larraceleta. Pre-Textos. Valencia.

Edelman, M. R. 1999. Peasants against globalization. Rural social movements in Costa. Stanford University Press. Stanford.

Escobar, A. y S. Álvarez (eds.) I992. The making of social movements in Latin America: Identity, strategy, and democracy. Westview Press. Boulder; Oxford.

EspinOSA, M. A. I998. "Práctica social y emergencia armada en el Cauca". En M. L. Sotomayor (ed.). Modernidad, identidad y desarrollo. Instituto Colombiano de Antropología/Ministerio de Cultura/Colciencias. Bogotá.

Fabian, J. I983. Time and the Other. New York Press. Columbia University.

FALS Borda, O. [I986] 2002. Retorno a la tierra (Historia doble de la Costa 4). Universidad Nacional de Colombia-Banco de la República-El Áncora Editores. Bogotá.

FARDON, R. I990. "Localizing strategies: The regionalization of ethnographic accounts. General Introduction”. En R. Fardon (ed.). Localizing strategies. Regional traditions of ethnographic writing. Scottish Academic Press and Smithsonian Institution Press. Edinburgh y Washington.

FERrater-Mora, J. I994. Diccionario de filosofía. I. Ariel. Barcelona.

FriedmanN, J. I987. "An interview with Eric Wolf”. Current Anthropology. 28.

Geertz, C. I96r. "Studies in peasant life: Community and society". Biennial Review of Anthropology. 2.

HANNERZ, U. I986. "Theory in anthropology: Small is beautiful? The problem of complex cultures". Comparative Studies in Society and History. 28.

Holtzman, J. 2004. “The Local in the local”. Current Anthropology. 45.

Hornblower, S. y A. Spawforth (eds.). [I949] 2003. The Oxford Classical Dictionary. Oxford University Press. Oxford.

Jameson, F. I98I. The political unconscious: Narrative as a socially symbolic act. Cornell University Press. Ithaca.

- I996. Political unconscious. Narrativa as a socially symbolic act. Cornell University Press. Ithaca. 


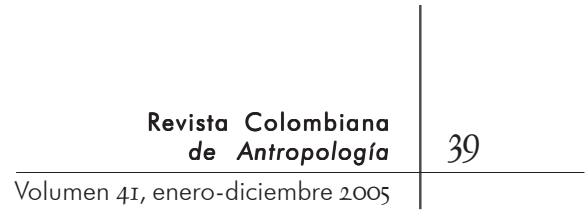

JENKINS, T. 1994. "Fieldwork and the perception of everyday life". Man. 29. New Series.

Kearney, M. 1996. Reconceptualizing the peasantry. Anthropology in global perspective. Westview Press. Oxford.

Latour, B. I988. The pasteurization of France. Harvard University Press. Cambridge.

LEWIS, O. [195I] I963. Life in a Mexican Village. Tepoztlan restudied. University of Illinois Press. Urbana.

Llobera, R. J. 1980. Hacia una historia de las ciencias sociales. Introducción. Editorial Anagrama. Barcelona.

MArcus, G. 1995. "Ethnography in/of the world system: The emergence of multi-sited ethnography". Annual Review of Anthropology. 24.

Marx, K. 1973. Las luchas de clases en Francia de I848 a I850. En Obras escogidas en tres tomos. Editorial Progreso. Moscú.

MAYER, E. 2002. The articulated peasant. Westview Press. Boulder.

MinTZ, S. 1998. "The localization of anthropological practice. From area studies to transnationalism. Critique Anthropology. I8.

- 2000. "Sow's ears and silver linings: A backward look at ethnography”. Current Anthropology. 4I.

Mol, A. y J. Law. 1994. "Regions, networks and fluids: Anaemia and social topology". Social Studies of Science. 24.

Nietzsche, F. 1994. Genealogía de la moral. M. E. Editores.

Ortega, H. 200I. "Demografía”. En G. B. Becerra y C. G. Valencia (eds). Historia, geografía y cultura del Cauca. Territorios posibles Universidad del Cauca. Popayán.

ORTNER, S. 1994. "Theory in anthropology since the sixties”. En N. B. Dirks et al. (eds.). Culture/Power/History. Princeton University Press. Princeton

Pelto, J. P. y G. Pelto. I978. Anthropological research. The structure of inquiry. Cambridge University Press. Cambridge.

Pletsch, E. C. I98I. "The three worlds, or the division of social scientific labor, circa I950-I975”. Comparative Studies in Society and History. 23.

Real Academia Española. I992. Diccionario de la lengua española I. Espasa Calpe. Madrid.

ReDFIELD, R. 1930. Tepoztlan: A Mexican Village. Chicago University Press. Chicago.

. 194I. The Folk Culture of Yucatan. Chicago University

Press. Chicago. 
Roseberry, W. I988. "Political economy". Annual Review of Anthropology. 17.

- 1989a. Anthropologies and histories. Essays in culture, history and political economy. Rutgers University Press. New Brunswick/Londres.

- I989b. "Peasants and the world". En S. Plattner (ed.). Economic anthropology. Stanford University Press. Stanford.

- 1997. "Marx and anthropology". Annual Review of Anthropology. 26.

Ruiz, C. A. 200I. "Cultivos ilícitos y etnicidad indígena”. En G. B. Becerra y C. G. Valencia (eds.). Historia, geografía y cultura del Cauca. Territorios posibles. Universidad del Cauca. Popayán.

Sahlins, M. 1976. Culture and practical reason. The University of Chicago Press. Chicago/Londres.

Scotт, J. 1996. "Social networks”. En A.K.a.J. Kuper. (ed.). The Social Science Encyclopaedia. Routledge. Londres/Nueva York.

Silverman, S. 1979. "The peasant concept in anthropology". The Journal of Peasant Studies. 7.

Singleton, V. y M. Mike. 1993. "Actor-Networks and ambivalence: General practitioners in the UK cervical screening programme". Social Studies of Science. 23.

SKIDMORE, E. T. 1998. "Studying the history of Latin America: A case of hemispheric convergence". Latin American Research Review. 33.

Stacul, J. 2000/200I. "Crossing the 'hidden frontier. Conversations with Eric R. Wolf”. Cambridge Anthropology. 22.

Steward, J. I953. "Culture patterns of Puerto Rico". Annals of the American Academy of Political and Social Science. January.

Tocancipá, J. I998. "Los estudios campesinos en la antropología colombiana. 1940-1960". Revista Problemas Políticos Latinoamericanos. 3 .

200I. "Región y mundos campesinos". En G. B. Becerra y C. G. Valencia (eds.). Historia, geografía y cultura del Cauca. Territorios posibles. Universidad del Cauca. Popayán.

Tocancipé-Falla, J. 2004. "Movimientos sociales, cultura política y poder regional. El caso del Movimiento del Macizo Colombiano (MMC)". Latin American Anthropology.

Vygotsky, L. S. I962. Though and Language (trans.) E.a.T.b.E.H.a.G. Vakar. The M.I.T. Press, Massachussets Institute for Technology/ John Wiley and Sons. Nueva York/Londres. 


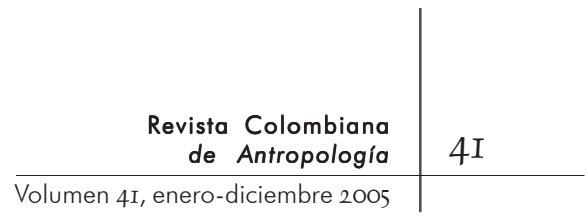

Wagley, C. y M. Harris. I955. "A Typology of Latin American subcultures". American Anthropologist. 57.

Wallerstein, I., C. Jumaa, E. F. Keller, J. Kocka, D. Lecourt, V. Y. Mudimbe, J. T. Peter, M.-R. Trouillot y R. Lee (eds.). ig96. Abrir las ciencias sociales. Comisión Gulbenkian para la estructuración de las ciencias sociales. Siglo XXI Editores. México.

Weber, M. 1944. Economía y sociedad. Economía y sociedad. I. Teoría de la organización social. Fondo de Cultura Económica. México.

Williams, R. I976-I983. Keywords. A vocabulary of Culture and Society. Fontana Press. Londres.

Wolf, E. 1955. "Types of Latin American peasantry". American Anthropologist. 57.

- 1957. "Closed corporate peasant communities in Mesoamerica and Central Java". Southwestern Journal of Anthropology. I3. 1986. "The vicissitudes of the closed corporate peasant community". American Ethnologist. I3. 1987a. Europa y la gente sin historia. Fondo de Cultura Económica. México. México. I987b. Las luchas campesinas del siglo XX. Siglo XXI.

Recibido: Io de mayo de 2004.

Aprobado: 29 de marzo de 2005. 